\title{
Performance Evaluation Approach for Multi-Tier Cloud Applications
}

\author{
Arshdeep Bahga, Vijay K. Madisetti \\ Electrical and Computer Engineering, Georgia Institute of Technology, Atlanta, USA. \\ Email: arshdeep@gatech.edu,vkm@gatech.edu \\ Received January $11^{\text {th }}, 2013$; revised February $9^{\text {th }}$, 2013; accepted February $17^{\text {th }}, 2013$
}

\begin{abstract}
Complex multi-tier applications deployed in cloud computing environments can experience rapid changes in their workloads. To ensure market readiness of such applications, adequate resources need to be provisioned so that the applications can meet the demands of specified workload levels and at the same time ensure that service level agreements are met. Multi-tier cloud applications can have complex deployment configurations with load balancers, web servers, application servers and database servers. Complex dependencies may exist between servers in various tiers. To support provisioning and capacity planning decisions, performance testing approaches with synthetic workloads are used. Accuracy of a performance testing approach is determined by how closely the generated synthetic workloads mimic the realistic workloads. Since multi-tier applications can have varied deployment configurations and characteristic workloads, there is a need for a generic performance testing methodology that allows accurately modeling the performance of applications. We propose a methodology for performance testing of complex multi-tier applications. The workloads of multi-tier cloud applications are captured in two different models-benchmark application and workload models. An architecture model captures the deployment configurations of multi-tier applications. We propose a rapid deployment prototyping methodology that can help in choosing the best and most cost effective deployments for multi-tier applications that meet the specified performance requirements. We also describe a system bottleneck detection approach based on experimental evaluation of multi-tier applications.
\end{abstract}

Keywords: Performance Modeling; Synthetic Workload; Benchmarking; Multi-Tier Applications; Cloud Computing

\section{Introduction}

Provisioning and capacity planning is a challenging task for complex multi-tier applications such as e-Commerce, Business-to-Business, Banking and Financial, Retail and Social Networking applications deployed in cloud computing environments. Each class of applications has different deployment configurations with web servers, application servers and database servers.

Over-provisioning in advance for such systems is not economically feasible. Cloud computing provides a promising approach of dynamically scaling up or scaling down the capacity based on the application workload. For resource management and capacity planning decisions, it is important to understand the workload characteristics of such systems, measure the sensitivity of the application performance to the workload attributes and detect bottlenecks in the systems. Performance testing of clouds applications can reveal bottlenecks in the system and support provisioning and capacity planning decisions. With performance testing it is possible to predict application performance under heavy workloads and identify bottlenecks in the system so that failures can be prevented. Bottlenecks, once detected, can be resolved by provisioning additional computing resources, by either scaling up systems (instances with more computing capacity) or scaling out systems (more instances of same kind).

In our previous work [1] we proposed the Georgia Tech Cloud Workload specification language (GT-CWSL) that provides a standard way for defining application workloads in a form that can be used by synthetic workload generation techniques, and a synthetic workload generator that accepted GT-CWSL workload specifications. In [1], we also described benchmark and workload models for describing different benchmarks in the form of building blocks.

In this paper we propose, 1) automated performance evaluation methodology for multi-tier cloud applications, 2) a rapid deployment prototyping methodology that can help in choosing the best and most cost effective deployments for multi-tier applications, 3) an architecture model that captures deployment configurations of multitier applications, 4) system bottleneck detection approach 
based on experimental evaluation of multi-tier applications. We have implemented the proposed approaches for performance evaluation, deployment prototyping and bottleneck detection in a set of tools that we named as the Georgia Tech Cloud Application Tester (GT-CAT).

In Section 2 we discuss related work. In Section 3, we describe the proposed methodology for performance evaluation of multi-tier cloud applications. In Section 4 we describe the experiment setup used demonstrate the proposed approaches for performance evaluation, deployment prototyping and bottleneck detection. In Section 5, we provide an experimental evaluation study of a multi-tier e-Commerce benchmark application on different deployment architectures.

\section{Related Work}

There are several workload generation tools developed to study web applications such as SPECweb99 [2], SURGE [3], SWAT [4] and HP LoadRunner [5]. Such workload generation tools repeatedly send requests from machines configured as clients to the intended systems under test. Table 1 provides a comparison of few workload generation tools. Several other tools generate synthetic workloads through transformation (e.g. permutation) of empirical workload traces [6-8]. Several studies on analysis and modeling of web workloads have been done [9-11]. Since obtaining real traces from complex multi-tier systems is difficult, a number of benchmarks have been developed to model the real systems [12-14].

Figure 1 shows a workflow used by traditional performance evaluation approaches, which require a real user to interact with the application to record scripts that are used by load generators. Tools such as HP LoadRunner [5] are based on the workflow shown in Figure 1. Figure 2 shows the proposed workflow for performance evaluation of multi-tier cloud applications. The proposed workflow is described in detail in Section 3.

We now describe the key differences between the traditional and proposed approaches.

\subsection{Capturing Workload Characteristics}

In traditional approach, such as in HP LoadRunner, to capture workload characteristics, a real user's interactions with a cloud application are first recorded as virtual user scripts. The recorded virtual user scripts then are parameterized to account for randomness in application and workload parameters. There is no underlying statistical model involved in such approaches as recorded scripts are used to drive the load generators. In the proposed approach, real traces of a multi-tier application which are logged on web servers, application servers and database servers are analyzed to generate benchmark and workload models that capture the cloud application and

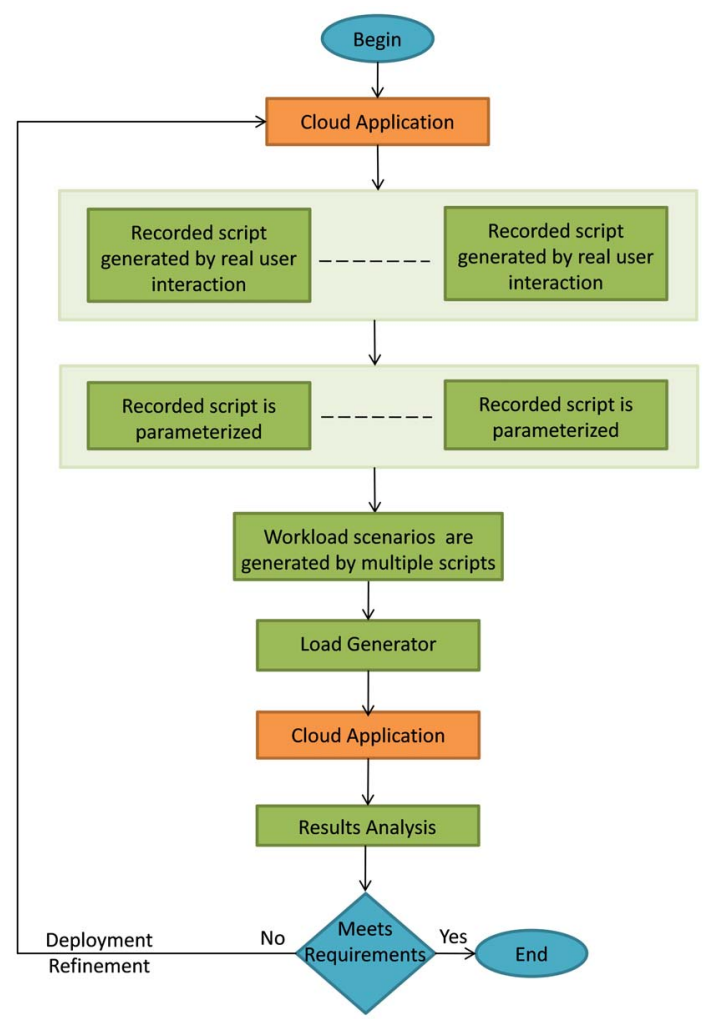

Figure 1. Traditional performance evaluation workflow based on a semi-automated approach.

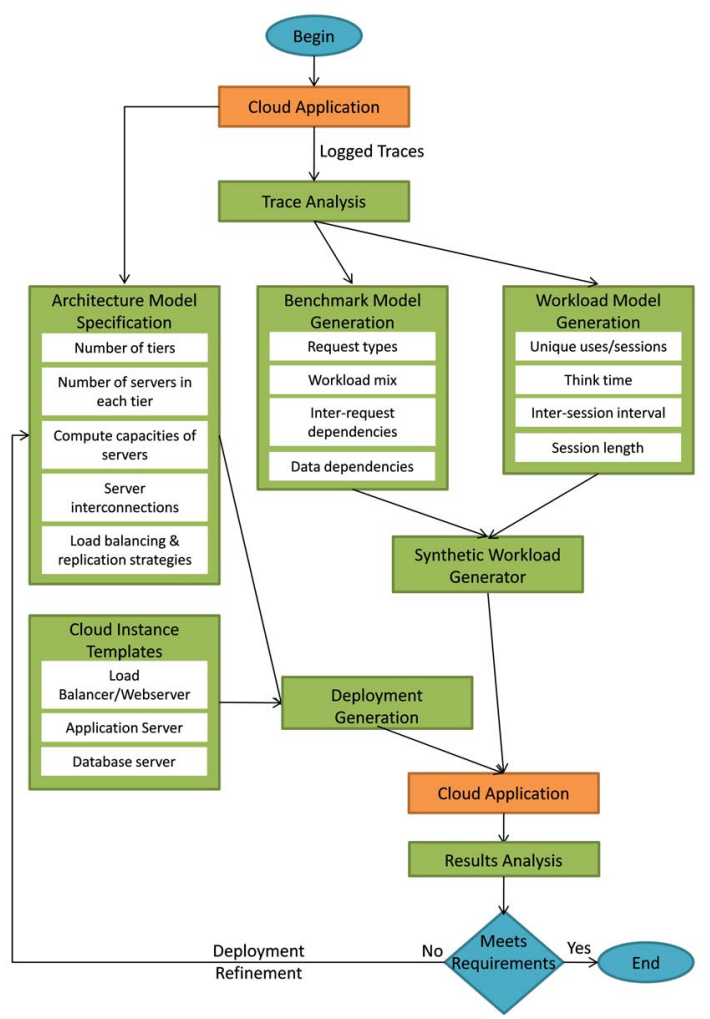

Figure 2. Proposed performance evaluation workflow based on a fully automated approach. 
Table 1. Comparison of related work.

\begin{tabular}{|c|c|c|c|c|}
\hline Reference & Approach & Application & Input/Output & Models used \\
\hline $\begin{array}{l}\text { SURGE } \\
\text { [3] }\end{array}$ & $\begin{array}{l}\text { Uses an offline trace generation engine } \\
\text { to create traces of requests. Web } \\
\text { characteristics such as file sizes, } \\
\text { request sizes, popularity, temporal } \\
\text { locality, etc are statistically modeled. }\end{array}$ & $\begin{array}{l}\text { Request generation for } \\
\text { testing network and } \\
\text { server performance }\end{array}$ & $\begin{array}{l}\text { Input-Pre-computed } \\
\text { data-sets consisting of the } \\
\text { sequence of requests to be } \\
\text { made, the number of } \\
\text { embedded files in each web } \\
\text { object to be requested, and the } \\
\text { sequences of Active and } \\
\text { Inactive OFF times to be } \\
\text { inserted between request. } \\
\text { Output-Synthetic workload } \\
\text { that agrees with six } \\
\text { distributional models. }\end{array}$ & $\begin{array}{l}\text { Six distributional models } \\
\text { make up the SURGE } \\
\text { model (file sizes, request } \\
\text { sizes, popularity, } \\
\text { embedded references, } \\
\text { temporal locality, and OFF } \\
\text { times). }\end{array}$ \\
\hline $\begin{array}{l}\text { SWAT } \\
\text { [4] }\end{array}$ & $\begin{array}{l}\text { Uses a trace generation engine that } \\
\text { takes sessionlets (a sequence of } \\
\text { request types from a real system user) } \\
\text { as input and produces an output trace } \\
\text { of sessions for stress test. SWAT uses } \\
\text { httperf for request generation. }\end{array}$ & $\begin{array}{l}\text { Stress testing } \\
\text { session-based } \\
\text { web applications }\end{array}$ & $\begin{array}{l}\text { Input-Trace of sessionlets } \\
\text { obtained from access logs of a } \\
\text { live system under test, } \\
\text { specifications of think time, } \\
\text { session length, session } \\
\text { inter-arrival time, etc. } \\
\text { Output-Trace of sessions for } \\
\text { stress test. }\end{array}$ & $\begin{array}{l}\text { Workload model used that } \\
\text { consists of attributes such } \\
\text { as session inter-arrival } \\
\text { time, session length, think } \\
\text { time, request inter-arrival } \\
\text { time and workload mix. }\end{array}$ \\
\hline $\begin{array}{l}\text { HP Load } \\
\text { Runner } \\
\text { [5] }\end{array}$ & $\begin{array}{l}\text { Based on empirical modeling } \\
\text { approach. A browser based Virtual } \\
\text { User Generator is used for interactive } \\
\text { recording and scripting. Scripts are } \\
\text { generated by recording activities of a } \\
\text { real user interaction with the } \\
\text { application. }\end{array}$ & $\begin{array}{l}\text { Performance testing of } \\
\text { web applications. }\end{array}$ & $\begin{array}{l}\text { Input-Load generators take } \\
\text { the virtual user scripts as } \\
\text { input. Output-Synthetic } \\
\text { workloads. }\end{array}$ & $\begin{array}{l}\text { Empirical modeling } \\
\text { approach used. Recorded } \\
\text { scripts are parameterized } \\
\text { to account for randomness } \\
\text { in application and } \\
\text { workload parameters. }\end{array}$ \\
\hline GT-CAT & $\begin{array}{l}\text { Based on analytical modeling } \\
\text { approach. Benchmark and workload } \\
\text { models are generated by analysis of } \\
\text { real traces of the application. Synthetic } \\
\text { workload generator generates } \\
\text { workloads based on the specifications } \\
\text { captured in models. }\end{array}$ & $\begin{array}{l}\text { Performance testing of } \\
\text { multi-tier cloud } \\
\text { applications. }\end{array}$ & $\begin{array}{l}\text { Input-Logged traces of real } \\
\text { application. Output-Synthetic } \\
\text { workload that has the same } \\
\text { workload characteristics as } \\
\text { real workloads. }\end{array}$ & $\begin{array}{l}\text { Benchmark, Workload \& } \\
\text { Architecture models used. }\end{array}$ \\
\hline
\end{tabular}

workload characteristics. A statistical analysis of the user requests in the real traces is performed to identify the right distributions that can be used to model the workload model attributes. In Section 3, we describe the benchmark and workload models in detail.

\subsection{Automated Performance Evaluation}

In traditional approach, multiple scripts have to be recorded to create different workload scenarios. This approach involves a lot of manual effort. In order to add new specifications for workload mix and new requests, new scripts need to be recorded and parameterized.

Writing additional scripts for new requests may be complex and time consuming as inter-request dependen cies need to be take care of. In the proposed approach, real traces are analyzed to generate benchmark and workload models. Various workload scenarios can be created by changing the specifications of the workload model. New specifications for workload mix and new requests can be specified by making changes in the benchmark model. This approach is faster as compared to traditional approach in which multiple virtual user scripts have to be recorded and parameterized to generate various workload scenarios. The benchmark and workload models drive the synthetic workload generator. The proposed performance evaluation methodology automates the entire performance evaluation workflow right from capturing user behavior into workload and benchmark models to generating synthetic workloads which have the same characteristics as real workloads.

\subsection{Realistic Workloads}

Traditional approaches which are based on manually generating virtual user scripts by interacting with a cloud application, are not able to generate synthetic workloads which have the same characteristics as real workloads. Although the traditional approaches allow creation of various workload scenarios using multiple recorded virtual user scripts, however, these workload scenarios are generally over simplifications of real-world scenarios in which a very large number of users may be simultaneously interacting with a cloud application. In the proposed approach, since real traces from a cloud application are used to capture workload and application characteristics into workload and benchmark models, the generated synthetic workloads have the same characteristics 
as real workloads. By statistical analysis of the user requests in the real traces, the proposed approach is able to identify the right distributions that can be used to model the workload model attributes such as think time, inter-session interval and session length.

\subsection{Rapid Deployment Prototyping}

Traditional approaches do now allow rapidly comparing various deployment architectures. Based on the performance evaluation results, the deployments have to be refined manually and additional virtual user scripts have to be generated with new deployments. In the proposed approach, an architecture model captures the deployment configurations of multi-tier applications. In Section 3.6, we describe a rapid deployment prototyping methodology that helps in choosing the best and most cost effecttive deployments for multi-tier applications that meet the specified performance requirements. With the proposed methodology, complex deployments can be created rapidly, and a comparative performance analysis on various deployment configurations can be accomplished.

\section{Proposed Methodology}

Figure 2 shows the proposed workflow for performance evaluation of multi-tier cloud applications. We now describe the steps in the performance evaluation workflow.

\subsection{Trace Analysis}

Figure 3 shows the benchmark and workload models generation process by analysis of logged traces of a cloud application. Real traces of a multi-tier application which are logged on web servers, application servers and database servers, have information regarding the user, the requests submitted by the user and the time-stamps of the requests. Each entry in the trace has a time-stamp, request type, request parameters and user's IP address. The trace generated from a benchmark has all the requests from all users merged into a single file. The trace analyzer identifies unique users/sessions based on the IP address or thread-ID from which the request came. The terms user and session cannot be always used interchangeably because a single user can create multiple sessions. Therefore, we use a time-threshold to identify a session. All requests that come from a single user within that threshold are considered as a single session.

\subsection{Benchmark Model}

The benchmark model includes attributes such as operations, workload mix, inter-request dependencies and data dependencies. The benchmark model captures the different requests types/operations allowed in the benchmark application, proportions of different request types and the

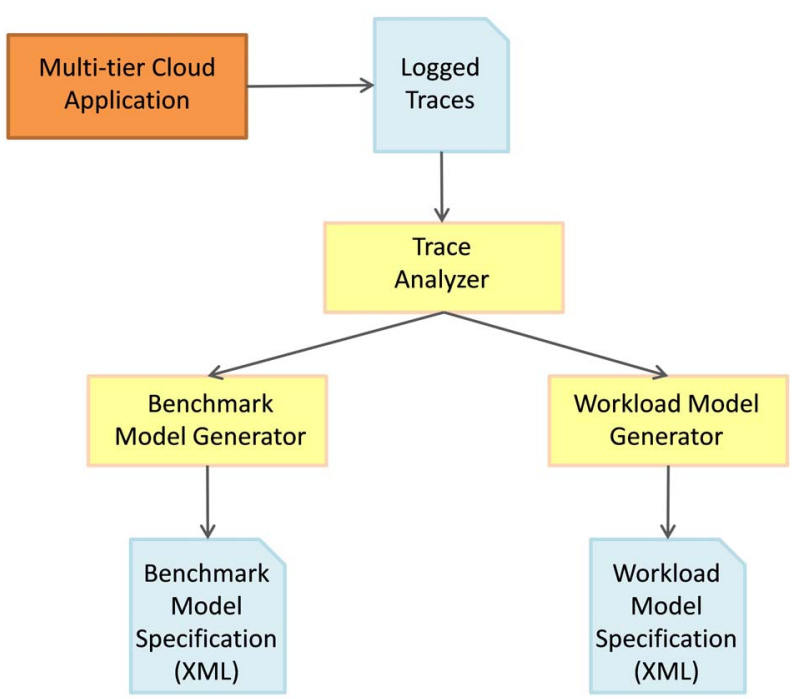

Figure 3. Benchmark and workload models generation by analysis of logged traces of cloud application.

dependencies between the requests. The benchmark model describes the semantic behavior of the requests. The semantic behavior determines the requests types of the application and the data associated with the requests. In our previous work [1] we described in detail the methodology used to in characterization of benchmarkmodel attributes which involves identification of different operations/request types in a benchmark application, proportions of different request types, i.e. the workload mix, the inter-request and data dependencies.

\subsection{Workload Model}

The workload model includes attributes of the workload such as inter-session interval, think time and session length. The workload model describes the time behavior of the user requests. The time behavior determines how many simultaneous requests are accepted by an application. When multiple users submit requests to an application simultaneously the workload model attributes such as inter-session interval, think time and session length are important to study the performance of the application. Think time and session length capture the client-side behavior in interacting with the application. Whereas the inter-session interval is a server-side aggregate, that captures the behavior of a group of users interacting with the application. For characterizing the workload model attributes, it is necessary to identify independent users/sessions in the trace. The trace analyzer identifies unique users and sessions from the trace of a benchmark application. A statistical analysis of the user requests is then performed to identify the right distributions that can be used to model the workload model attributes such as inter-session interval, think time and session length. The methodology adopted in characterizing workload model 
attributes is described in detail in our previous work [1].

\subsection{Architecture Model}

Figure 4 shows a multi-tier deployment generation process using cloud instance templates and architecture model specifications. Architecture model includes specifications for all the tiers in the deployment. To provide a modular approach for creating complex multi-tier deployments, we created cloud instance templates for load balancer, web server, application server and database server. Cloud instance templates include a base Linux image (CentOS or Ubuntu) and a set of startup scripts that install and configure the software (such as HAProxy load balancer, Apache web server, PHP application server, MySQL database server, etc.). Additional startup scripts are used for deploying an application on the deployment specified in the architecture model. The instance size for each tier (computing capacity) is specified in the architecture model. Complex deployments can have can have multiple instances of the same type in each tier. For simplicity in describing multi-tier deployment configurations we use the naming convention- $\# L$ (size)/\#A (size)/\#D (size)), where $\# L$ is the number of instances running load balancers and web servers, $\# A$ is the number of instances running application servers, $\# D$ is the number of instances running database servers and (size) is the size of an instance. Specifications for the number of instances for each tier are included in the architecture model. The advantage of using a separate architecture model is that the performance evaluations become independent of application under study. With architecture model and cloud instance templates, complex deployments can be created rapidly, which allows evaluating the performance of an application on various deployment architectures. Deployments can be rapidly scaled up (vertical scaling) or scaled out (horizontal scaling) by making changes in the architecture model.

\subsection{Synthetic Workload Generation}

Figure 5 shows the synthetic workload generation process based on benchmark and workload model specifications. The synthetic workload generator is built using the Faban run execution and management infrastructure [15], which is an open source facility for deploying and running benchmarks. We have extended the Faban Harness to accept GT-CWSL specifications that are generated by the GT-CWSL code generator using the benchmark and workload models. This synthetic workload generator allows generating workloads for multi-tier cloud applications that are deployed across several nodes in a cloud. The Master agent contains a web-server that runs the GT-CAT web interface which is used to launch and queue performance test runs and visualize the results. Run

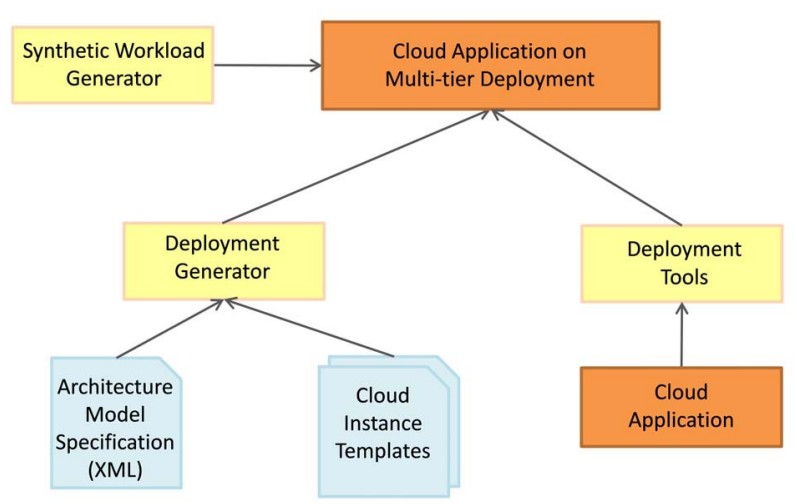

Figure 4. Multi-tier deployment generation using cloud instance templates and architecture model specifications.

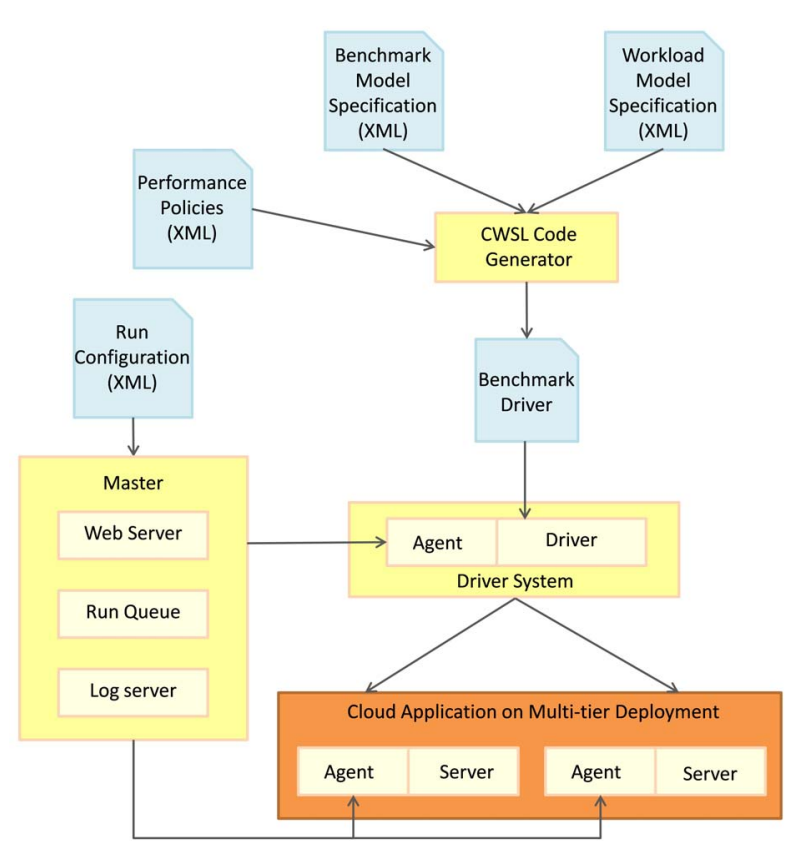

Figure 5. Synthetic workload generation based on benchmark and workload model specifications.

Queue manages the performance test runs which are run in a first in first out (FIFO) manner. Log Server collects pseudo real time logs from the systems under test. Agents are deployed on both the driver systems and the systems under test. These agents control the performance runs and collect the system statistics and metrics which are used for performance evaluation. Multiple agent threads are created by an agent, where each thread simulates a single user. Registry registers all the agents with the Master so that the master can submit the load driving tasks to the agents. The logic for workload generation, workload characteristics, application operations and the logic for generating requests and the associated data for each of the operations are specified in the Driver. Run configuration provides the input parameters that control the performance test run on a multi-tier cloud application. 
Run configuration contains specifications of the ramp up, steady state and ramp down times, the number of users, output directory, etc. The performance policies include a series of service level objectives (SLO's) that define the performance metrics such as the response time specification for each request in the application.

\subsection{Deployment Prototyping}

Though from the standpoint of a user, the cloud computing resources should look limit-less, however due to complex dependencies that exist between servers in various tiers, applications can experience performance bottlenecks. Deployment prototyping can help in making deployment architecture design choices. By comparing performance of alternative deployment architectures, deployment prototyping can help in choosing the best and most cost effective deployment architecture that can meet the application performance requirements.

Given the performance requirements for an application, the deployment design is an iterative process that involves the following steps:

1) Deployment Design: Create the deployment with various tiers as specified in the deployment configuration and deploy the application.

2) Performance Evaluation: Verify whether the application meets the performance requirements with the deployment.

3) Deployment Refinement: Deployments are refined based on the performance evaluations. Various alternatives can exist in this step such as vertical scaling, horizontal scaling, etc.

\subsection{Bottleneck Detection}

Traditional approaches for bottleneck detection in multitier systems have used average resource utilization values for bottleneck analysis. However, complex-multi-tier cloud applications can experience non-stationary workloads. Average values fail to capture stochastic non-stationary seasonality in workloads. Therefore, we use kernel density estimates for bottleneck detection. A probability density estimate of the data is computed based on a normal kernel function using a window parameter that is a function of the number of data points. Kernel density estimates indicate the percentage of time a resource spent at a particular utilization level. In Section 3.7, we demonstrate the bottleneck detection approach with three set of experiments with different deployment architectures.

\section{Experiment Setup}

To demonstrate the proposed approaches for performance evaluation, deployment prototyping and bottleneck detection, we used the Rice University Bidding System [13] benchmark. RUBiS is an auction site prototype which has been modeled after the internet auction website eBay. We used a PHP implementation of RUBiS for the experiments. For measuring system statistics, we used systat and collectd utilities. To study the effect of different deployment configurations of the application performance we performed a series of experiments by varying the architecture model and the application deployment configurations. The experiments were carried out using the Amazon Elastic Compute Cloud (Amazon EC2) instances. For the experiments we used small (1 EC2 compute unit), large (4 EC2 compute units) and extralarge (8 EC2 compute unit) instances, where each EC2 compute unit provides an equivalent CPU capacity of $1.0-1.2$ GHz 2007 Opteron processor or 2007 Xeon processor.

\section{Results}

We instrumented the PHP implementation of the RUBiS benchmark application and obtained the traces of the user requests. From the analysis of the logged traces the benchmark and workload models were generated. In the first set of experiments we used a $1 L$ (large)/2A(small) $/ 1 D$ (small) configuration and varied the number of users from 400 to 2800 . For these experiments we used ramp up and ramp down times of 1 minute and steady state time of 10 minutes.

Figure 6(a) shows the CPU usage density of one of the application servers. This plot shows that the application server CPU is non-saturated resource. Figure 6(b) shows the database server CPU usage density. From this density plot we observe that the database CPU spends a large percentage of time at high utilization levels for more than 2400 users. Figure 6(c) shows average CPU utilizations of one of the application servers and the database server. This plot also indicates that the database server experienced high CPU utilization whereas the application server CPU was is non-saturated state. Figure 6(d) shows the density plot of the database server disk I/O bandwidth. This plot shows a bimodal shape of the disk I/O bandwidth density curve. From a thorough analysis of Figure 6(b), we observe a slight bimodality in the shape of the database CPU utilization curve for more than 1500 users. This bimodality in Figures 6(b) and (d) occurs due to the long read/write requests. When the database server is servicing a long read/write request, the CPU utilization remains low while it is waiting for the $\mathrm{I} / \mathrm{O}$.

Figure 6(e) shows the density plot of the network out rate for one of the application servers. Figure 6(f) shows the average throughput and response time. A strong correlation is observed between the throughput and average application server network out rate. Throughput continuously increases as the number of users increase from 400 to 2400. Beyond 2400 users, we observe a decrease 


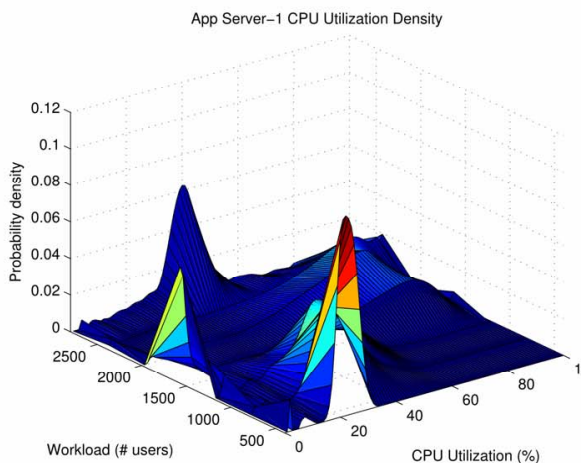

(a)

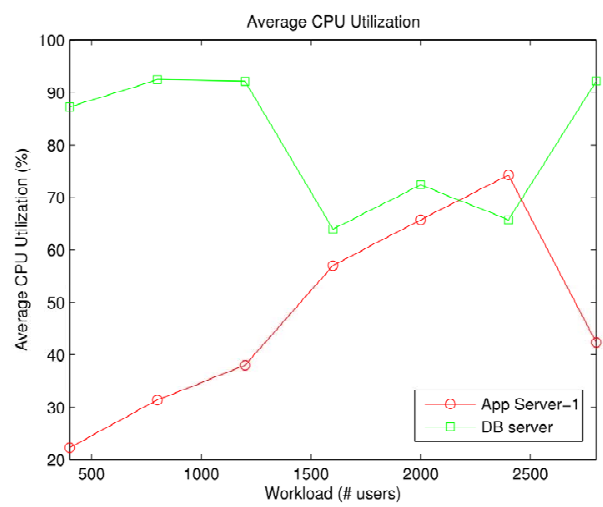

(c)

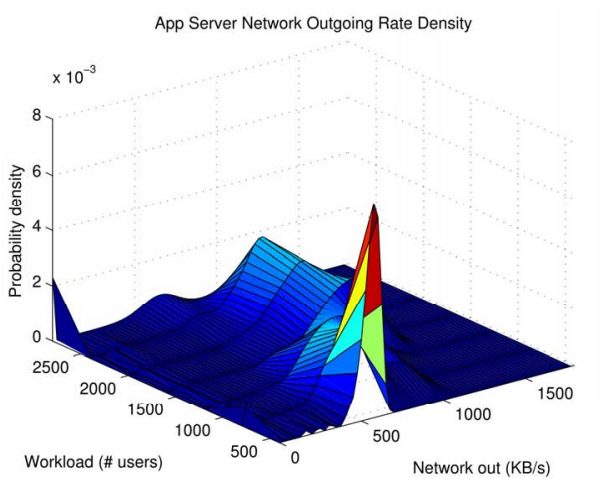

(e)

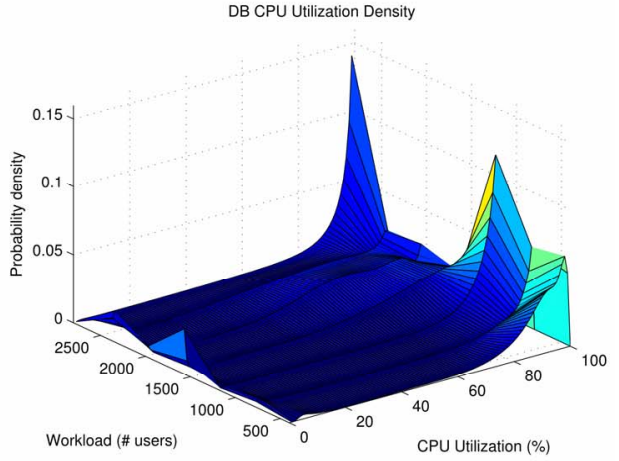

(b)

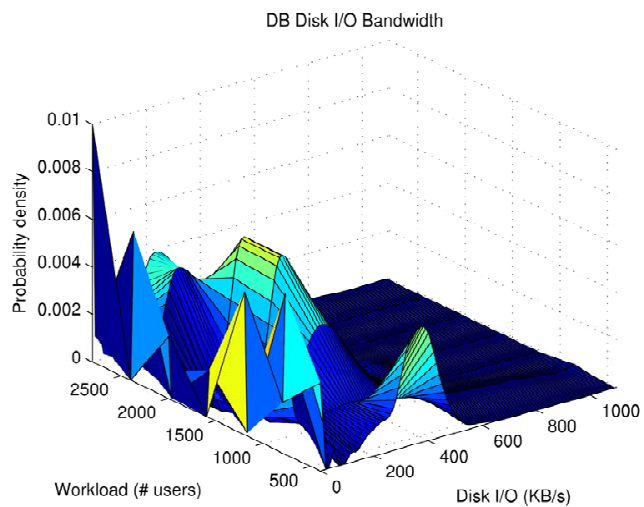

(d)

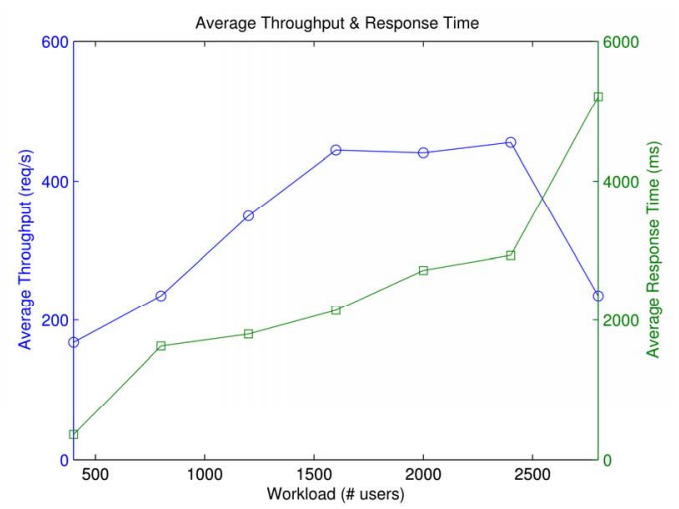

(f)

Figure 6. (a) App server-1 CPU usage density; (b) DB CPU usage density; (c) Average CPU utilization; (d) DB disk I/O bandwidth; (e) App server network outgoing rate; (f) Average throughput and response time.

in throughput, which is due to the high CPU utilization density of the database server CPU. From the analysis of density plots of various system resources we observe that the database CPU is a system bottleneck.

\subsection{Scale-Up Experiments}

The proposed deployment prototyping methodology allows rapidly and elastically changing application deployments using the architecture model and the cloud instance templates. To demonstrate this capability, we performed a second set experiments by scaling up the deployment configuration used in the first set of experiments. In the second set of experiments we used a $1 L($ xlarge $) / 2 A($ small $) / 1 D($ xlarge $)$ configuration and varied the number of users from 400 to 2800.

Figure 7(a) shows the CPU usage density of one of the application servers. Unlike in the first set of experiments where the application server CPU was a nonsaturated resource, in this set, we observe that the application server CPU spends a large percentage of time at high utilization levels. Figure 7(b) shows the database 
server CPU usage density. From this plot we observe that the database server CPU is a non-saturated resource. Figure 7(c) shows average CPU utilizations of one of the application servers and the database server. This plot also indicates that the application server experienced high CPU utilization whereas the database server CPU was in a non-saturated state.

Figure 7(d) shows the average throughput and response time. Comparing throughput and response timeplots of the first and second set of experiments we observe that the maximum throughputs in both set of experiments are very similar. However, the response times in the second set of experiments are lower than those in the first set, which is due to the higher compute capacities of the load balancer, web server and database server in the second set as compared to the first set.

Comparing results of first and second set of experiments, we observe that system bottleneck shifts from database CPU in first set to application server CPU in the second set. Scaling-up the deployment configuration from $1 L($ large $) / 2 A($ small $) / 1 D($ small $)$ to $1 L($ xlarge $) / 2 A$ (small)/1D(xlarge), does not result in increase in throughput, however lower response times are observed with the scaled-up deployment.

\subsection{Scale-Out Experiments}

We performed a third set experiments by scaling out the deployment configuration used in the first set of experiments. In the third set of experiments we used a $1 L$ (xlarge $) / 3 A($ small $) / 1 D$ (xlarge) configuration and varied the number of users from 400 to 2800 .

Figure 8(a) shows the CPU usage density of one of the application servers. We observe that the application server CPU spends a large percentage of time at high utilization levels for more than 2000 users. Figure 8(b) shows the database server CPU usage density. From this plot we observe that the database server CPU is a non-saturated resource. Figure 8(c) shows average CPU utilizations of one of the application servers and the database server. This plot also indicates that the application server experienced high CPU utilization whereas the database server CPU was in a non-saturated state. Figure 8(d) shows the average throughput and response time. Comparing throughput and response time plots of the second and third set of experiments we observe that the

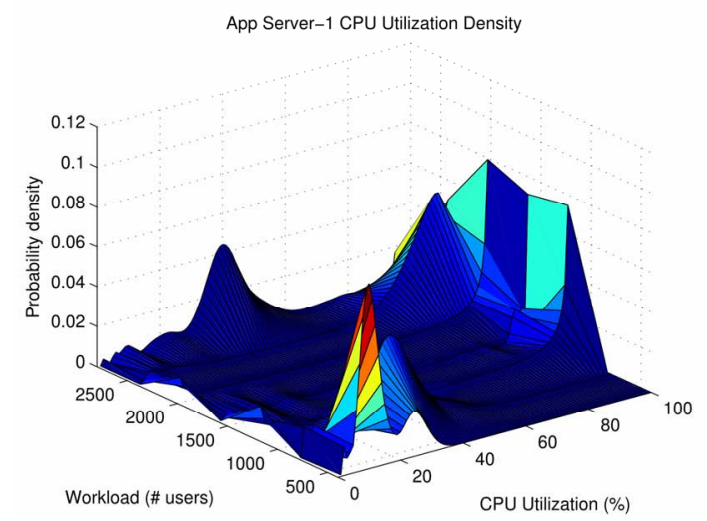

(a)

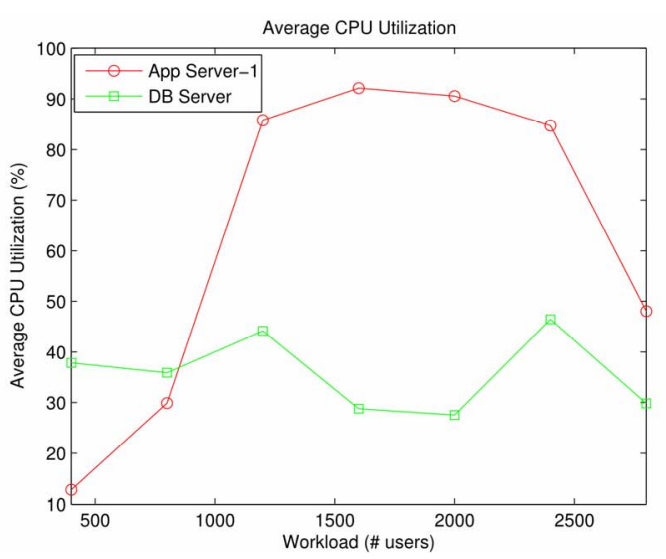

(c)

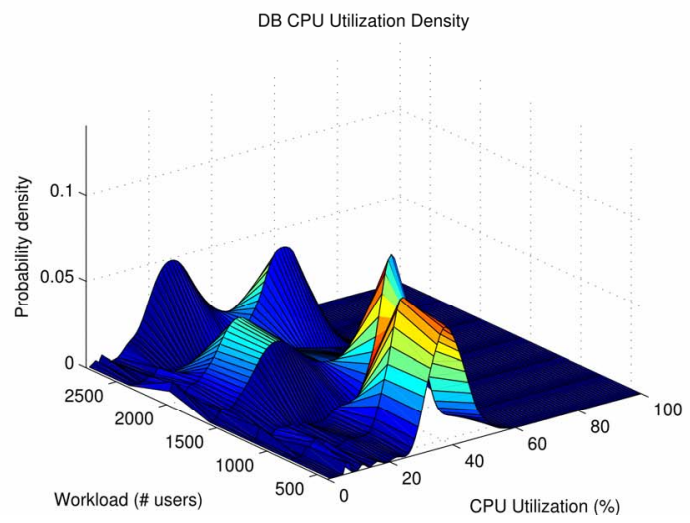

(b)

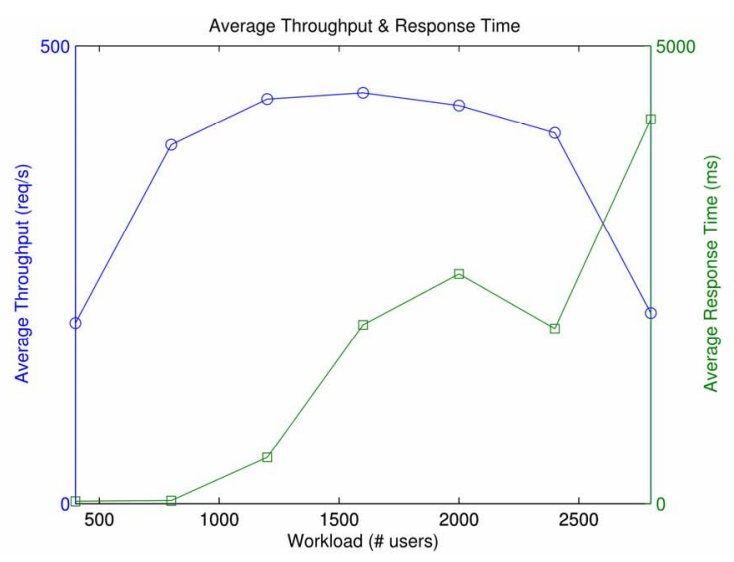

(d)

Figure 7. Scale-up experiment: (a) App server-1 CPU usage density; (b) DB CPU usage density; (c) Average CPU utilization; (d) Average throughput and response time. 
App Server-1 CPU Utilization Density

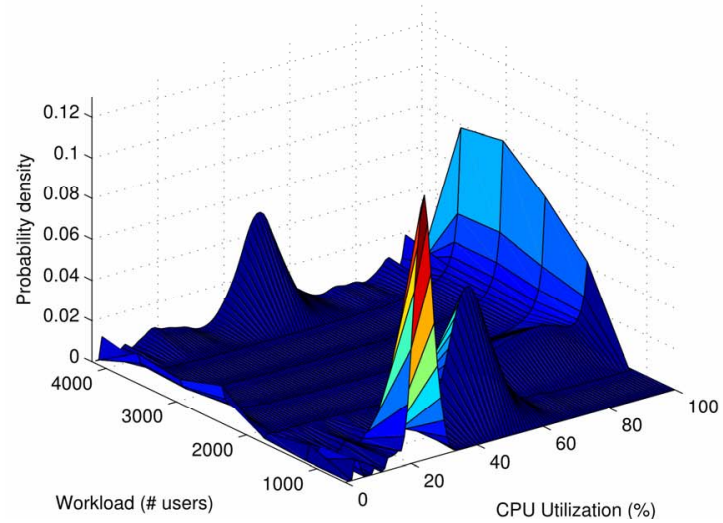

(a)

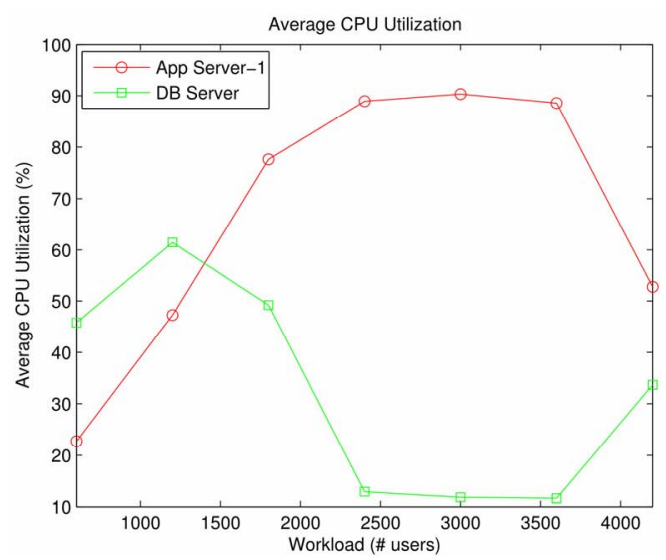

(c)
DB CPU Utilization Density

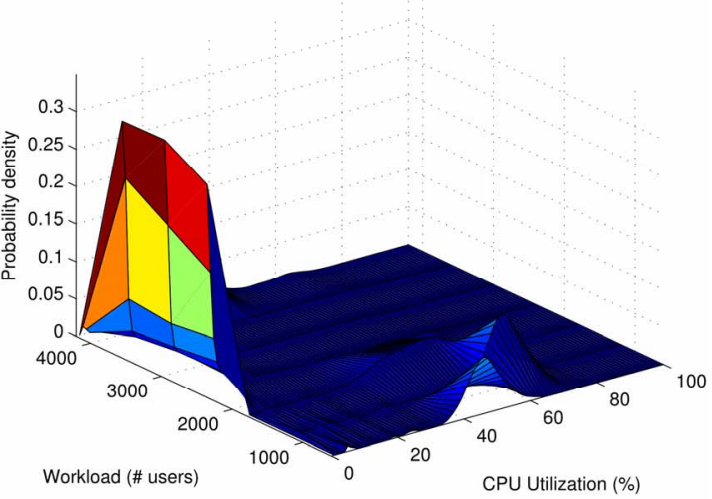

(b)

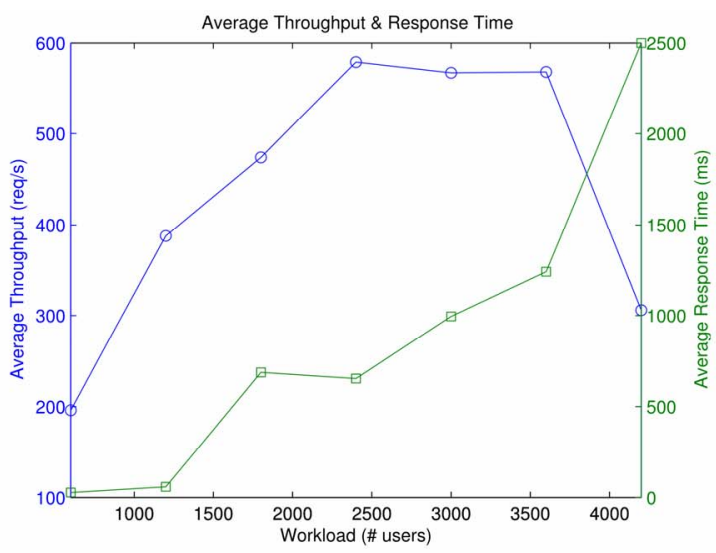

(d)

Figure 8. Scale-out experiment: (a) App server-1 CPU usage density; (b) DB CPU usage density; (c) Average CPU utilization; (d) Average throughput and response time.

maximum throughput in the third set is more than the second set. Moreover, slightly lower response times are observed in the third set as compared to the second set.

Comparing results of second and third set of experiments, we observe that scaling-out the deployment configuration from $1 L($ xlarge $) / 2 A($ small $) / 1 D($ xlarge $)$ to $1 L$ (xlarge)/3A(small)/1D(xlarge), results in an increase in throughput and decrease in response times.

\section{Results Interpretation}

In this section we provide a general interpretation of the results shown in Section 5 and also provide design guidelines for multi-tier deployments architectures. There are several factors that should be considered before designing multi-tier deployments architectures:

1) Performance requirements: Performance requirements are typically specified in the service level agreements (SLA) which provide response time or throughput requirements for each request type (or web page) in the application. Before designing a multi-tier deployment, a careful understanding of the performance requirements is required. The proposed deployment prototyping approach can help in making the right choices for deployment architectures. From results in Section 5 we observe that throughput increases as the number of users submitting requests to an application increase and eventually becomes relatively constant and may even drop due to system bottlenecks. The maximum throughput is limited by system bottlenecks such as high CPU utilizations of servers in various tiers, database disk I/O bandwidth, etc.

2) Workload Characteristics: Performance of multitier cloud applications can be highly sensitive to the characteristics of workloads. Insights into characteristics of application workloads can help in making the right design choices for deployment architectures. For example, an application that has database read intensive workloads, can benefit from a database cluster that services the read requests [16]. For read intensive workload, distributed memory object caching systems such as Memcached servers can also speed up the application performance [17]. Applications with database read/write intensive workloads, can benefit from high memory and high CPU capacity cloud instances. Characterization of workload 
attributes such as session length, inter-session interval, think-time, workload mix, etc. by analysis of logged traces of applications, can help in getting insights into the workload characteristics.

3) Cost: From the results in Section 5, we observed that both horizontal and vertical scaling can help in improving application performance. Both types of scaling options involve additional costs either for launching additional servers or provisioning servers with higher memory and compute capacities. The proposed deployment prototyping approach can help in rapidly comparing deployments with both types of scaling options. Thus, with deployment prototyping the most cost-effective deployment architecture can be chosen.

4) Complexity: A simplified deployment architecture can be more easier to design and manage. Therefore, depending on application performance and cost requirements, it may be more beneficial to scale vertically instead of horizontally. For example, if equivalent amount of performance can be obtained at a more cost-effective rate, then deployment architectures can be simplified using small number of large server instances (vertical scaling) rather than using a large number of small server instances (horizontal scaling).

\section{Conclusion}

In this paper, we describe a generic performance evaluation methodology for complex multi-tier applications deployed in cloud computing environments. The proposed methodology captures multi-tier application workloads and deployment architectures in three separate models-benchmark model, workload model and architecture. The advantage of using three separate models to capture workload characteristics and deployment architectures is that the performance evaluation process becomes independent of application under study. Results show that with the proposed deployment prototyping and bottleneck detection approaches it is possible to rapidly compare different deployment architectures and detect system bottlenecks, so that the right design choices can be made for deployment architectures.

\section{REFERENCES}

[1] A. Bahga and V. K. Madisetti, "Synthetic Workload Generation for Cloud Computing Applications,” Journal of
Software Engineering and Applications, Vol. 4, No. 7, 2011, pp. 396-410. doi:10.4236/jsea.2011.47046

[2] SPECweb99, 2012.

http://www.spec.org/osg/web99

[3] P. Barford and M. E. Crovella, "Generating Representative Web Workloads for Network and Server Performance Evaluation,” SIGMETRICS, Vol. 98, 1998, pp. 151160.

[4] D. Krishnamurthy, J. A. Rolia and S. Majumdar, "SWAT: A Tool for Stress Testing Session-Based Web Applications," Proceedings of International CMG Conference, Dallas, 7-12 December 2003, pp. 639-649.

[5] H. P. LoadRunner, 2012. http://www8.hp.com/us/en/software/software-product.htm l?compURI=tcm:245-935779

[6] A. Mahanti, C. Williamson and D. Eager, "Traffic Analysis of a Web Proxy Caching Hierarchy," IEEE Network, Vol. 14, No. 3, 2000, pp. 16-23.

doi:10.1109/65.844496

[7] S. Manley, M. Seltzer and M. Courage, “A Self-Scaling and Self-Configuring Benchmark for Web Servers," Proceedings of the ACM SIGMETRICS Conference, Madison, 22-26 June 1998.

[8] Webjamma, 2012. http://www.cs.vt.edu/ chitra/webjamma.html,

[9] G. Abdulla, "Analysis and Modeling of World Wide Web Traffic,” Ph.D. Thesis, Chair-Edward A. Fox, 1998.

[10] M. Crovella and A. Bestavros, "Self-Similarity in World Wide Web Traffic: Evidence and Possible Causes, IEEE/ ACM Trans,” Networking, Vol. 5, No. 6, 1997, pp. 835846. doi:10.1109/90.650143

[11] D. Mosberger and T. Jin, "httperf: A Tool for Measuring Web Server Performance,” ACM Performance Evaluation Review, Vol. 26, No. 3, 1998, pp. 31-37. doi:10.1145/306225.306235

[12] D. Garcia and J. Garcia, "TPC-W E-Commerce Benchmark Evaluation,” IEEE Computer, 2003.

[13] RUBiS, 2012. http://rubis.ow2.org

[14] TPC-W, 2012. http://jmob.ow2.org/tpcw.html

[15] Faban, 2012. http://faban.sunsource.net

[16] 2012. http://www.mysql.com/products/cluster

[17] 2012. http://memcached.org 